\title{
Evaluation des Integrationspotenzials von Massen- medien - theoretische und methodische Überlegungen
}

\section{Philomen Schönhagen}

Der Beitrag knüpft an Ausführungen Otfried Jarrens zu den Leistungen bzw. dem Potenzial der Massenmedien im Hinblick auf eine "Integrationskommunikation" (vgl. Heft 1/2000) an und entwickelt einige theoretische sowie forschungspraktische Gedanken zur empirischen Analyse solcher Leistungen. Dazu werden Jarrens Überlegungen in einem knappen Überblick zusammengefasst und in Bezug zu einem Theorieansatz gesetzt, der sich im Hinblick auf das Problem gesellschaftlicher Integration durch Medien, wie es von Jarren dargelegt wird, als außerordentlich fruchtbar erweist. Unter anderem können damit die Funktionen von "intermediären Einrichtungen" wie Parteien, Verbänden etc. als Kommunikationsteilnehmer einerseits sowie der Massenmedien als Kommunikationsvermittler andererseits deutlich gekennzeichnet werden. Weiter folgen daraus methodische Überlegungen im Sinne des von Jarren geforderten „erweiterten Modells" für die empirische Analyse des massenmedialen Integrationspotenzials. Es werden konkrete Teilfragen für die empirische Analyse abgeleitet sowie eine erweiterte Inhaltsanalyse vorgestellt, die hier Erfolg versprechend eingesetzt werden könnte.

\section{Zentrale Gedanken Otfried Jarrens zur gesellschaftlichen Integration durch Medien}

Ausgangspunkt der Argumentation Jarrens ist die Feststellung, dass Medien „im Zusammenhang mit anderen Organisationen, vor allem in der Interaktion mit den intermediären Akteuren der Gesellschaft (wie Parteien, Verbänden, Kirchen, Gewerkschaften, Neuen Sozialen Bewegungen) die Aufgabe zu[kommt], gesellschaftliche Selbstverständigungsdiskurse zu ermöglichen und zu führen“" (Jarren 2000: 22/23; Hervorhebungen P. S.). Diese Sichtweise ähnelt der von der Systemtheorie formulierten Funktion der Massenmedien, gesellschaftliche Selbstbeobachtung zu ermöglichen (vgl. Luhmann 1996: 173). Im Übrigen kann diese Funktion der Massenmedien bzw. des Journalismus als Vermittler derartiger gesellschaftlicher Diskurse auch historisch, gewissermaßen als Motiv für die Entstehung autonomer Medien und des Journalismus nachgewiesen werden (vgl. Schönhagen 1998, u. a. S. 291ff.).

Jarren versteht Integration als einen Prozess, „der Einzelne, Gruppen wie Organisationen umfasst, der sich anhaltend auf unterschiedlichen gesellschaftlichen Ebenen vollzieht und daher aufgrund seines hohen Komplexitätsgrades als soziales Totalphänomen nicht hinreichend empirisch gemessen oder erfasst werden kann. Integration als Konstruktion sozialer Realität vollzieht sich im Wesentlichen durch Kommunikation" (2000: 23; Hervorhebung P. S.). Diese Feststellung entspricht dem so genannten „kommunikativen Prinzip“, nach dem sich Gemeinschaft bzw. Gesellschaft und Kommunikation wechselseitig bedingen, ja konstituieren, sowie den Überlegungen der Wissenssoziologie, etwa von Berger/Luckmann und Alfred Schütz zur Konstruktion gesellschaftlicher Wirklichkeit (vgl. dazu sowie zu Folgerungen für die Funktion des Journalismus Schönhagen 1999). Ganz im Sinne auch solcher Ansätze folgert Jarren: „Da die gesellschaftliche Kommunikation in der modernen Gesellschaft sich weitgehend über Medien vollzieht, kommt den Massenmedien eine zentrale Funktion für (Integrations-)Diskurse (als Vermittler) und auch als soziostruktureller Infrastrukturfaktor zu“ (2000: 23; Hervor- 
hebung P. S.); dies gilt in besonderer Weise, aber nicht ausschließlich, für den öffentlichrechtlichen Rundfunk. Jarren präzisiert den Zusammenhang zwischen gesellschaftlicher Kommunikation und Integration, indem er herausstellt, dass Erstere „gleichermaßen symbolische wie materielle Integration“ ermögliche, denn: „Im Ergebnis kann es zu gemeinsam geteilten Wissensbeständen, zu spezifischen sozialen Handlungsformen [man könnte ergänzen: gemeinsamen Normen und Werten; P. S.], zur Bildung von Organisationen sowie zur Herausbildung von Institutionen kommen, die den jeweiligen Bedürfnissen nach Integration materiell wie auch symbolisch entsprechen" (ebd.: 25). Einschränkend hält Jarren fest, dass gesellschaftliche „Einheit“ gewissermaßen nur in Form von kommunizierten Konzepten existiert und „der ständigen Interpretation - konkurrierender Kommunikationen - unterliegt. Wenn also die Einheit der Gesellschaft weder erreicht noch (empirisch) sicher festgestellt werden kann, so kann doch über Kommunikation Einheit und Integration in bestimmten Räumen, für ausgewählte Gruppen und in bestimmten zeitlichen Phasen erzeugt werden" (ebd.: 31$)$.

Jarren spricht aber nicht nur von den Medien als Vermittlern der gesellschaftlichen Diskurse, sondern bezeichnet auch die so genannten „intermediäre[n] Einrichtungen“ wie Parteien, Verbände etc. als deren „Vermittlungsinstanzen“ (ebd.: 25). Diese „intermediären Einrichtungen" seien jedoch zugleich Akteure der besagten Diskurse: „(...) für diese Kommunikation bedarf es der Akteure und entsprechender Vermittler. $\mathrm{Zu}$ den Akteuren zählen vorrangig die gesellschaftsweit agierenden Organisationen, die wir dem intermediären Bereich zurechnen können. Und relevante Vermittler sind vor allem jene Organisationen, wie wir sie in Form eigenständig agierender Medien kennen“ (ebd.: 31). Hier müssten meiner Ansicht nach die Funktionen besagter intermediärer Einrichtungen bzw. Akteure einerseits und der Medien andererseits präziser gefasst und unterschieden werden. Tatsächlich handelt es sich um zwei unterschiedliche Phänomene mit zumindest weitgehend unterschiedlichen Funktionen, für deren Verständnis es hilfreich ist, den Aspekt der Kommunikationsrepräsentanz einzubeziehen (siehe weiter unten, Punkt 2).

Sodann markiert Jarren wichtige Aspekte im Hinblick auf die empirische Forschung zum Thema Integration und Medien, wobei er auch die neueren Entwicklungen des Mediensystems einbezieht. Zu beachten seien bei der Analyse die Unterschiede der Medien „bezüglich ihres Potenzials zur gesellschaftlichen Integration, was sich an Organisationsstrukturen, Angebotsformen, -inhalten und -qualitäten empirisch nachweisen lässt" (ebd.: 38). Im vorliegenden Zusammenhang ist vor allem interessant, dass es nach Jarren für solche Analysen „eines erweiterten Modells [bedarf]: Der empirische Blick allein auf die Medien, ihre Inhalte oder die Rezeption reicht nicht aus. Themen und Wissen, auch Kenntnisse über Normen, Werte und Verfahren, werden zwar gesellschaftsweit weitgehend über Medien vermittelt, aber nicht von ibnen (allein) erzengt. Dies geschieht durch Akteure in allen Teilsystemen, wesentlich aber über jene Akteure, die dem intermediären System zuzurechnen sind. Sie sind es, die vor allem die Themen für die Medienvermittlung bereitstellen und damit weitgehend die öffentliche Agenda bestimmen. Medien greifen auf die bereitgestellten Informationen zu, weniger greifen sie eigenständig Themen auf. (...) Die Interaktionen zwischen Akteuren, Medien und Publikum gewinnen damit für die Analyse an Bedeutung, denn in diesen Prozessen wird auch über die Möglichkeiten einer Integrationskommunikation entschieden. In empirischer Forschung ist demnach diesen Interaktionen und ihren Ergebnissen, auch und gerade im Hinblick auf das Leistungsprofil von einzelnen Medien (Grad an Vermittlungsleistung vs. Eigenleistung), Aufmerksamkeit zu schenken“ (ebd.; Hervorhebungen P. S.). Dabei sei die „empirische Sichtweise (...) um normative Aspekte (...) zu ergänzen: (...) An die 
einzelne Medienorganisation kann dabei aber nicht ein konkreter Integrationsauftrag, sondern nur ein Auftrag zur Abbildung relevanter Selbstverständigungsdiskurse gerichtet werden. Das bedeutet, Medien haben die Diskurse gesellschaftlicher Akteure zu berücksichtigen und sie werden auf Offenheit, Vielfalt und Pluralität verpflichtet" (ebd.: 38/39; Hervorhebungen P. S.). ${ }^{1}$

\section{Ein „erweitertes Modell“ vermittelter gesellschaftlicher Kommunikation}

Eine kommunikationstheoretische Basis der dargestellten Überlegungen Jarrens sowie für das von ihm geforderte „erweiterte Modell“ für eine empirische Analyse kann ein Modell bzw. ein Theorieansatz bereitstellen, der in der deutschen Kommunikationswissenschaft schon lange existiert, bedauerlicherweise jedoch bislang zu wenig gewürdigt $^{2}$ oder vorschnell als vermeintlich normativ abgelehnt wurde ${ }^{3}$ : die Münchner Theorie sozialer Kommunikation und das daraus hervorgegangene Modell der Massenkommunikation (vgl. u. a. Wagner 1995a; einen kurzen Überblick bietet Schönhagen 1998: 263 - 268). Hans Mathias Kepplinger betonte unlängst, dass dieser Ansatz „mehr Beachtung verdient" (2000: 429).

Mit diesem Theorieansatz lässt sich die oben geforderte Präzisierung des Vermittlungsbegriffs bzw. der Rollen und Funktionen der intermediären Einrichtungen einerseits und der Massenmedien andererseits problemlos leisten. Dafür sind besonders zwei Aspekte des Münchner Ansatzes entscheidend: 1. die analytische Trennung von Vermittlungs- und Kommunikationsprozess und 2. die Berücksichtigung des Phänomens der Kommunikations-Repräsentanz, die für das Verständnis sozialer Kommunikation grundlegend ist. ${ }^{4}$

1 Interessanterweise entspricht diese normative Forderung der Selbstverpflichtung vieler „Zeitungsmacher" oder Journalisten in Deutschland seit dem Aufkommen der periodischen Zeitungen im 17. Jahrhundert und weit bis ins 19. Jahrhundert hinein vor allem in der lokalen Presse, ebenso wie bei den amerikanischen Kolonialzeitungen in der ersten Hälfte des 18. Jahrhunderts, die unter dem Begriff der Unparteilichkeit bzw. impartiality verbreitet war und sich in einem ebenso differenzierten wie praktisch bewährten journalistischen Vermittlungskonzept äußerte. Vgl. dazu Schönhagen 1998.

2 Ausnahmen sind u. a. Wolfgang R. Langenbucher und Peter Glotz, die verschiedentlich und mit durchaus kritischem Blick auf Vorteile und praktische Relevanz dieses Ansatzes hingewiesen haben. Vgl. etwa Glotz 1990: 253/254, Langenbucher 1996a, 1996b: 8/9 u. 1997: 29. Interessant ist, dass außerdem Martin Stock das Konzept im Zusammenhang mit rundfunkrechtlichen Fragen kritisch diskutiert und als Basis herangezogen hat (vgl. 1985: 43ff.). Neuerdings hat Roland Burkart seine Überlegungen zum Konzept eines „diskursiven Journalismus“ ausdrücklich an den Münchner Theorieansatz angeknüpft (vgl. 1998: 169f.).

3 Vgl. jüngst Kohring 2000: 253. Bei näherer Betrachtung des Theorieansatzes zeigt sich jedoch, dass er zunächst, wie viele andere Theorieansätze oder Modelle auch, funktionale Erklärungen und Unterscheidungen liefert, aus denen allerdings normative Folgerungen durchaus abgeleitet werden können.

4 Im Zusammenhang mit Fragen der Öffentlichkeit wurde inzwischen auch im Rahmen anderer Ansätze bemerkt, dass man ohne Berücksichtigung von Repräsentanz-Phänomenen bzw. Repräsentanten nicht auskommt: Vgl. die Beiträge von Friedhelm Neidhardt und Jürgen Gerhards in Neidhardt (Hrsg.) (1994). In der Münchner Theorie spielt dieser Aspekt eine zentrale Rolle und ist entsprechend ausgearbeitet (vgl. Wagner 1995b: 32ff.). 


\subsection{Massenmedien als Vermittlungsinstanzen sozialer Kommunikation: der Vermittlungsprozess}

Unter anderem anhand einer historischen Betrachtung (vgl. Wagner 1980) lässt sich zeigen, dass die so genannten Massenmedien aus dem Bedürfnis heraus entstanden sind, in komplexen Gesellschaften eine umfassende soziale Kommunikation zu gewährleisten, trotz räumlicher und zeitlicher Distanzen der Kommunikationsteilnehmer. ${ }^{5}$ Technische Medien allein reichen jedoch zur Realisierung einer gesellschaftsweiten Kommunikation nicht aus, jedenfalls dann nicht, wenn diese auch für alle Gesellschaftsmitglieder überschaubar sein soll. Eine solche Überschaubarkeit erfordert die Reduktion von Komplexität, welche nur mit redaktionellen Vermittlungsleistungen, also mittels zusätzlicher personaler Vermittler realisierbar ist. ${ }^{6}$ Massenmedien werden hier folglich nicht in einem rein technischen Sinn, sondern als Vermittlungssysteme verstanden; Entsprechendes gilt für den Vermittlungsbegriff, der neben der notwendigen technischen Verbreitung (durch Signalproduktion und Distribution) vor allem die redaktionelle Aufbereitung, d. h. die mediengerechte Konzentration (Selektion und Transformation) des gesellschaftlichen Mitteilungs- und Kommunikationsgeschehens umfasst (vgl. Wagner 1980: 6). ${ }^{7}$

5 In weniger komplexen Gesellschaften konnte bzw. kann die gesellschaftsweite Kommunikation im Modus der Versammlung realisiert werden (vgl. Wagner 1995b: 17f.). Unter sozialer Kommunikation verstehe ich hier, in Anlehnung an Hans Wagner (vgl. 1978: 205, dort nur die erste Teildefinition), die je aktuelle öffentliche $-d$. h. prinzipiell für alle potenziellen Kommunikationspartner bzw. Partner-Kollektive, -Gruppen etc. offene - gesellschaftliche Kommunikation, sowohl die partikulare, also auf der Ebene von Teilsystemen stattfindende, als auch die gesamtgesellschaftliche Kommunikation (universelle Massenmedien). Teilweise wird, abweichend von dem hier zugrunde gelegten Verständnis, in der Münchner Theorie der Begriff der sozialen Kommunikation enger gefasst und nur auf die gesamtgesellschaftliche Ebene bezogen (vgl. ebd., zweite Teildefinition). „Im allerweitesten Sinn ist ,Soziale Kommunikation' [jedoch auch; P. S.] bei Aswerus das aktuelle Gesellschaftsgespräch überhaupt“ (Wagner 1993: 184; vgl. dazu auch Aswerus 1993: 84ff.). Eine eingehendere Begriffsdiskussion (auch allgemein des Kommunikationsbegriffs sowie des Medien- und Massenmedien-Begriffs) kann hier aus Platzgründen leider nicht stattfinden, ist aber Bestandteil des in Arbeit befindlichen Habilitationsprojekts der Verfasserin.

6 Inwieweit diese Funktion möglicherweise durch intelligente Software, etwa die so genannten intelligenten Agenten erbracht werden könnte, ist eine andere Frage, die hier jedoch nicht diskutiert werden kann. Auch dabei gilt es aber, den Aspekt der Repräsentanz zu beachten, auf den ich noch zu sprechen komme.

7 Vermittlung ist also nicht im Sinne eines bloßen Transports von Informationen (etwa im Sinne einer Transportmetapher, welche z. B. Krippendorf 1994: 85/86 kritisiert) oder eines Verlautbarungsjournalismus misszuverstehen. Ebenso wenig ist Vermittlung von „Informationen über die Welt" gemeint. Auch in diesem Sinne wird in der Literatur bisweilen der Vermittlungsbegriff gebraucht bzw. kritisiert; vgl. z. B. Hartmann 1996: 107. Hier geht es dagegen um Kommunikations-Vermittlung. Dabei sind die Medienorganisationen bzw. die darin tätigen Journalisten auch nicht in dem Sinne passiv, dass sie nur solche Mitteilungen vermitteln würden, die von außen an sie herangetragen werden. Es ist eine ihrer wichtigen Funktionen, aktiv durch Recherche solche potenziellen Kommunikationsteilnehmer (seien es Einzelne oder Gruppen) zu ermitteln und in die Vermittlungsleistungen einzubeziehen, die nicht von sich aus an die Medien herantreten, etwa weil sie über keinerlei Organisations- und Repräsentanzstrukturen, keine Sprecher, Pressestellen etc. verfügen oder aus anderen Gründen nicht aktiv in das Kommunikationsgeschehen eingreifen. 
Die Funktion der Massenmedien bzw. der darin tätigen Journalisten beschränkt sich natürlich nicht nur auf diese aktive Vermittlungsleistung, sondern wird ergänzt um eigene Kommunikationsbeiträge, sei es in Form von Kommentaren, Glossen, Satiren, Reportagen u. Ä., Literatur-, Film- und Theaterkritiken oder Gesprächsbeiträgen von Show- und Talkmastern, die über die Moderationsfunktion hinausgehen. Bisweilen fungieren Journalisten auch als „Sprachrohre“ anderer Kommunikationsteilnehmer, die aus unterschiedlichen Gründen nicht selbst an die Öffentlichkeit treten können. Für das Zustandekommen und Funktionieren (gesamt-)gesellschaftlicher Kommunikation ist allerdings die Rolle der Medieninstitutionen als aktive Vermittler grundlegend und unverzichtbar. Diese beiden Funktionen der Vermittlung sozialer Kommunikation einerseits und eigener Gesprächsbeiträge der Journalisten andererseits - die der so genannten Medium- und Faktorfunktion entsprechen, welche nach der Rechtsprechung des Bundesverfassungsgerichts den Massenmedien zukommt (vgl. Branahl 1996: 20) - müssen bei der Analyse von Integrationsleistungen als „Vermittlungs- versus Eigenleistung“ (vgl. Jarren 2000: 38) unbedingt unterschieden werden. Medien bzw. Journalisten vermitteln aber in der Regel weder ausschließlich noch vorrangig eigene Gesprächsbeiträge, sondern in erster Linie Beiträge anderer Kommunikationsteilnehmer, d. h. gesellschaftliche Kommunikationsprozesse, die von diesem Vermittlungsprozess zu unterscheiden sind.

Im Vermittlungsprozess sind die Massenmedien (bzw. die Journalisten sowie die technischen Mitarbeiter) demnach die Akteure, die übrige Gesellschaft fungiert als Ziel dieses Prozesses, als Publikum. ${ }^{8}$ Dieser reine Vermittlungsprozess wird in Modellen wie etwa dem von Gerhard Maletzke (1963) unter Einbeziehung diverser Feedback-Prozesse abgebildet (vgl. dazu auch Stock 1985: 32ff.); unberücksichtigt aber bleiben die Kommunikationsprozesse, welche den Gegenstand der Vermittlung darstellen.

\subsection{Zugrunde liegende soziale Kommunikationsprozesse und Kommunikations- repräsentanz}

Die gesellschaftliche Kommunikation spielt sich nun, anders als die Vermittlung, keineswegs zwischen Massenmedien bzw. Journalisten auf der einen und einem passiven Publikum bzw. Rezipienten auf der anderen Seite ab, sondern diverse gesellschaftliche Akteure oder Sprecher - im Münchner Theorieansatz Kommunikationspartner genannt - stehen wechselseitig miteinander in Kommunikation. Prinzipiell kann jedes Mitglied der Gesellschaft - also sowohl die in den Medien tätigen professionellen Vermittler als auch die Rezipienten der Medien - Kommunikationsteilnehmer sein und

8 In Bezug auf die Vermittlung ist das Publikum also tatsächlich passiv, sind die Rezipienten Empfänger, die mit den professionellen Vermittlern in der Regel nicht die Rollen tauschen können, von Ausnahmen wie bei Offenen Kanälen und Ähnlichem abgesehen. (Was nichts damit zu tun hat, dass der Rezeptionsprozess selbst natürlich aktive Selektion und Interpretation umfasst.) Wie im Weiteren gezeigt wird, sind die Rezipienten aber nicht auf diese Rolle im Vermittlungsprozess beschränkt, sondern sind zugleich am Kommunikationsprozess beteiligt. Dass sich der Vermittlungsprozess in einem Teil der computervermittelten Kommunikation, etwa bei Homepages, Chats und Newsgroups im World Wide Web anders darstellt, ist offensichtlich. Der entscheidende Unterschied ist, dass man es in diesen Fällen nicht mit Medieninstitutionen zu tun hat, welche über eine Redaktion verfügen. Bei einer Netzzeitung dagegen ist die Rollenverteilung im Vermittlungsprozess dieselbe wie bei den klassischen Massenmedien. 
dabei zwischen den Rollen eines Sprechers und eines Angesprochenen ständig wechseln. ${ }^{9}$

Tatsächlich treten als aktive Teilnehmer, d. h. Sprecher und Angesprochene im gesellschaftlichen Kommunikationsgeschehen, in den meisten Fällen vorwiegend Akteure aus dem von Jarren so genannten „intermediären Bereich“ in Erscheinung, etwa Politiker, Gewerkschaftsvorsitzende, Unternehmenssprecher und -vorsitzende, Verbandspräsidenten und dergleichen. Daraus kann jedoch nicht gefolgert werden, alle übrigen Gesellschaftsmitglieder seien am Kommunikationsprozess nicht beteiligt und es bliebe ihnen nur die Rezipientenrolle im Vermittlungsgeschehen, im Gegenteil: Man hat es hier mit Erscheinungen des Phänomens der Kommunikations-Repräsentanz zu tun. Die in den Medien auftretenden Sprecher äußern sich in den meisten Fällen nicht für sich allein, sondern repräsentieren Kollektive wie Institutionen, Organisationen, Vereine, Bürgerbewegungen etc. Dabei müssen sie nicht unbedingt formale Repräsentanten solcher Gruppen sein (bestellte Sprecher, Vorsitzende u.Ä.), auch wenn das bei den in den Medien vermittelten Sprechern häufig der Fall ist, sondern sie können ebenso für eine nicht näher bestimmbare, nicht organisierte Anzahl von Personen, welche sich mit den Äußerungen identifizieren, quasi automatisch mitsprechen. ${ }^{10}$ Auch aufseiten der Angesprochenen existieren selbstverständlich solche Gruppenzugehörigkeiten bzw. Repräsentanzbeziehungen (häufig werden in der Massenkommunikation ohnehin nicht Einzelne angesprochen, sondern Sprecher richten ihre Äußerungen an ganze Zielgruppen, eine Partei etwa), sodass es letztlich immer Kollektive von Kommunikationsteilnehmern sind, die medienvermittelt miteinander im Gespräch stehen. Man kann somit auch treffend von „Kommunikation im Plural“ reden (Wagner 1995a: 229).

Kommunikationsteilnehmer sind demnach nicht nur die kleinere Zahl von Gesellschaftsmitgliedern, die als aktive Sprecher und direkt (namentlich) Angesprochene auftreten, sondern auch der Großteil all derer, die - in themen- und zeitabhängig ständig wechselnden Konstellationen - zu der „Wir-Gruppe“ der je einen und anderen zu rechnen sind. Bei den einzelnen Kommunikationsprozessen kann es des Weiteren solche Personen (-Gruppen) geben, die weder direkt Sprecher oder Angesprochene noch von diesen Repräsentierte sind. Hans Wagner nennt diese zutreffend „latente Partner“ (vgl.

9 Wagner spricht in Anlehnung an Otto Groth von „Ausgangspartnern“ und „Zielpartnern“ als den ständig wechselnden Rollen der Kommunikationspartner (vgl. u. a. 1995a: 215). Ausgangspartner sind die Urheber von Mitteilungen, Zielpartner diejenigen, an welche die Mitteilungen gerichtet sind. Damit kennzeichnen diese Begriffe die Rollen wesentlich präziser als der Akteurs-Begriff, der ebenso Objekte (z. B. die Beteiligten an einem Unfall, über den berichtet wird) wie Subjekte der Kommunikation (z. B. den Polizeisprecher, der über den Unfall eine Mitteilung macht) sowie Subjekte der Vermittlung (s. o.) bezeichnen kann. Treffender ist die Bezeichnung „Sprecher“ für den Urheber von Mitteilungen, auch wenn letztere natürlich nur teilweise in gesprochener Form gemacht werden, sondern häufig schriftlich, als Pressemitteilungen, Berichte etc. vorliegen. Nachdem der Sprecher-Begriff mittlerweile im Fach gängig geworden ist, passe ich mich hier dem Sprachgebrauch an; als Pendant zum Sprecher wird der Begriff des „Angesprochenen“ eingeführt, da Kommunikation immer mindestens zweier Partner oder Teilnehmer bedarf. Der auch von Jarren selbstverständlich benutzte Begriff des Vermittlers entspricht der Terminologie des Münchner Ansatzes, ebenso wie der Rezipient, wobei unbedingt zu beachten ist, dass letzterer hier nicht eine Kommunikations-, sondern eine Vermittlungsrolle bezeichnet.

10 Wagner spricht hier von „statistischer Realrepräsentanz“ im Gegensatz zur formal legitimierten Realrepräsentanz (vgl. 1980: 13ff. sowie ders. 1978: 73ff.). 
1978: 83ff.), da sie prinzipiell jederzeit durch eigene Aktivität oder aufgrund journalistischer Recherche ebenfalls zu vermittelten Kommunikationspartnern bzw. -teilnehmern werden können. Das ist wegen der Periodizität der Berichterstattung häufig der Fall; jedoch können Meinungen, Wissensbestände etc. bestimmter Gruppen auch langfristig in der medienvermittelten Kommunikation latent bleiben.

\subsection{Folgerungen für die Analyse des medialen Integrationspotenzials}

Als entscheidende Erweiterung eines entsprechenden Analysemodells folgt daraus zunächst die Einbeziehung der Kommunikationsteilnehmer oder Sprecher als Urheber gesellschaftlicher Kommunikationsprozesse, welche von den Medien aufgegriffen und an die Gesellschaft zum Zwecke der Selbstbeobachtung bzw., wie Jarren treffend formuliert, der Selbstverständigung zurückvermittelt werden. Zudem muss berücksichtigt werden, dass vorwiegend Repräsentanten als aktive Kommunikationsteilnehmer auftreten, diese aber für Kollektive sprechen, welche somit als „repräsentierte Partner“ (vgl. Wagner 1978: 59ff.) zumindest indirekt ebenfalls am Kommunikationsprozess teilnehmen.

Die Unterscheidung von Kommunikations- und Vermittlungsprozess muss aber auch auf der Rezipientenseite systematisch weitergedacht werden: Dieselben Personen, die im Vermittlungsprozess Rezipienten sind, nehmen zugleich innerhalb des gesellschaftlichen Kommunikationsgeschehens bestimmte Rollen ein. Erst wenn man dies berücksichtigt, wird die Integrationsleistung der Medien vollständig sichtbar. Denn aus der Gesamtheit der Rezipienten, die ja den Großteil der Gesellschaftsmitglieder darstellen, stammen zugleich die Mehrzahl der aktiven und repräsentierten Kommunikationsteilnehmer; nicht aus den Reihen der professionellen Vermittler, auch wenn diese ebenfalls zugleich Rollen im Kommunikationsgeschehen wahrnehmen.

Diese gleichzeitige Wahrnehmung zweier Rollen in miteinander verschränkten Prozessen (man kann von „Rollen-Unionen“ sprechen; Wagner 1995a: 234) kann in folgenden Ausprägungen auftreten: Ein Rezipient ist zugleich Sprecher, der seine eigenen medienvermittelten Äußerungen rezipiert (z. B. Altkanzler Kohl, der einen Fernsehbericht über seine Aussagen vor dem Spenden-Untersuchungsausschuss sieht), oder Angesprochener (z. B. Bundeskanzler Schröder, der einen Zeitungsbericht über an ihn gerichtete Vorwürfe eines Oppositionssprechers zur Kenntnis nimmt), oder ein von einem der Kommunikationspartner Repräsentierter (im letzteren Beispiel entweder Mitglied bzw. Anhänger der Regierung oder der Opposition), oder latenter Kommunikationspartner (z. B. Vertreter einer weiteren, noch nicht vermittelten Ansicht); ein Journalist ist nicht nur Vermittler, sondern zugleich Sprecher (z. B. häufig in Kommentaren, Reportagen etc.), oder auch - wie bei Wagner nicht ausdrücklich angegeben - Angesprochener (was mit steigender Selbstbezüglichkeit der Medien immer häufiger vorkommt), seltener latenter Partner, da Vermittler zumeist ihren unmittelbaren Zugang zur Vermittlung nutzen, wenn sie einen Kommunikationsbeitrag leisten wollen. Im Übrigen sind die Vermittler natürlich immer zugleich Rezipienten des von ihnen selbst bzw. von anderen Medien Vermittelten.

Vor diesem Hintergrund lassen sich nun auch die Funktionen der von Jarren sowohl als Akteure wie auch als Vermittler angesprochenen intermediären Einrichtungen auf der einen und der Medien als Vermittlungsinstanzen auf der anderen Seite klar unterscheiden. Die intermediären Einrichtungen, also gesellschaftlichen Organisationen, Institutionen u. Ä., bzw. genauer deren Vertreter fungieren in der sozialen Kommunikation als Gesprächsteilnehmer, als Sprecher wie Angesprochene, die dabei immer Reprä- 
sentanten sind. Damit bündeln sie die Interessen vieler Einzelner und bringen diese gegenüber der Öffentlichkeit sowie anderen gesellschaftlichen Teilsystemen zur Geltung (was Jarren wohl als deren „vermittelnde“ Funktion anspricht). Neben diesen intermediären Einrichtungen können jedoch auch andere, weniger institutionalisierte Kollektive sowie Einzelne Sprecher und Angesprochene sein. Die Medienorganisationen dagegen greifen die Kommunikationsbeiträge dieser intermediären Einrichtungen sowie anderer Sprecher auf, bearbeiten sie redaktionell und vermitteln sie sodann an die Rezipienten (und damit u. a. auch an die Angesprochenen) bzw. je nach Medientyp an eine breite Öffentlichkeit bzw. bestimmte Teilöffentlichkeiten. Auch wenn sie diese Vermittlungsleistung um eigene Diskussionsbeiträge ergänzen - was man zutreffend als „Fremd-“ und „Eigenvermittlung“ unterscheiden kann (vgl. Wagner 1988: 187) -, kommt den so genannten Massenmedien damit die Rolle der eigentlichen Vermittlungsinstanzen im gesellschaftlichen Kommunikationsgeschehen $\mathrm{zu}$, wie auch Jarren konstatiert.

Nicht nur kommunikationstheoretisch, sondern auch im Hinblick auf die hier erörterte Frage der Integrationsfunktion der Medien ist es bedeutsam, die jeweiligen, ständig wechselnden Rollenunionen zu berücksichtigen. Denn dann geht es aus der Sicht des Medienpublikums nicht nur darum, im Sinne der Selbstbeobachtung der Gesellschaft einen möglichst umfassenden Überblick über die unterschiedlichen Standpunkte, Interessen, Wissensbestände etc. zu erlangen, die in der vermittelten Kommunikation zum Ausdruck kommen. Es geht darüber hinaus, wie Jarren deutlich macht, um Selbstverständigung der Gesellschaft bzw. derer Mitglieder und Teilsysteme untereinander: Die Rezipienten der massenmedial vermittelten Kommunikation stellen den Großteil der Gesellschaftsmitglieder dar bzw. bilden mit ihren Handlungen deren Teilsysteme, folglich ist eine zumindest kommunikative Integration nur dann gegeben, wenn sich die Rezipienten in den vermittelten Mitteilungen direkt oder repräsentiert als Kommunikationspartner wiederfinden. Unter kommunikativer Integration ist hier die Beteiligung an der sozialen Konstruktion von Wirklichkeit zu verstehen, welche ihrerseits nur über Kommunikation stattfinden kann. Wenn man davon ausgeht, dass sich Gesellschaft überhaupt erst durch Kommunikation konstituiert ${ }^{11}$, dann wird zumindest eine grundlegende gesellschaftliche Integration ${ }^{12}$ (nur) über die kommunikative Beteiligung an diesem Konstruktionsprozess realisiert (vgl. auch Jarren 2000: 23 u. 29ff.), sei es eine direkte Beteiligung als Sprecher und Angesprochene oder die indirekte Teilnahme als Repräsentierte, die weitaus häufiger vorkommt.

Zudem tragen die Massenmedien dadurch zur gesellschaftlichen Integration bei, dass Sprecher sich über Medien auch an ihre eigenen „Wir-Gruppen“ richten und damit Repräsentanzbeziehungen herstellen oder festigen. Nicht selten finden Kollektive erst aufgrund der Vermittlung von Äußerungen Einzelner organisiert zusammen, was wiederum ihre Chancen erhöht, in den Massenmedien vermittelt zu werden.

11 Diese These entspricht dem „kommunikativen Prinzip“, wonach Gemeinschaft durch Mitteilung konstituiert wird (vgl. Wagner 1993: 154ff.; Aswerus 1993: 18). Dies wurde unter Punkt 1 bereits angesprochen; vgl. dazu auch Marcic 1965: 165. Cornelia Bohn formuliert diese These in zugespitzter Form auf der Basis der Systemtheorie: „(...), daß Gesellschaft aus nichts anderem als Kommunikationen besteht" (1999: 16).

12 Kritisch zum Integrationsbegriff und seinen unterschiedlichen Bedeutungen vgl. Jarren 2000: 22ff.; Rühl 1985: 22ff.; Braun 1990: $25 \mathrm{ff}$. 


\subsection{Einzelfragen zur Analyse des medialen Integrationspotenzials}

Für die Analyse und Beurteilung der Integrationsleistungen von Massenmedien ist es demnach entscheidend, welche Sprecher bzw. Kollektive zu den verschiedenen Themen und wie diese jeweils mit ihren Âußerungen und Argumenten vermittelt werden. Dazu bieten die Überlegungen des Münchner Ansatzes nicht nur den theoretischen Hintergrund, sondern auch ganz spezifische Zugänge sowie eine entsprechend ausgearbeitete Methodik, welche im Anschluss noch erläutert wird (siehe Punkt 3).

Zunächst sind die jeweiligen Integrationsleistungen natürlich stark vom Medientyp abhängig, Begrenzungen ergeben sich dadurch von vornherein auf mehreren Ebenen: sachlich (z. B. Fachmedien, Special-Interest-Zeitschriften, Spartenkanäle versus themenuniverselle Tageszeitungen oder Vollprogramme), weltanschaulich (z. B. Parteimedien oder katholisches Kirchenblatt versus weltanschaulich universelle Medien) sowie geografisch (z. B. Lokal- versus überregionale Tageszeitung). ${ }^{13}$

Sodann stellen sich folgende Fragen: Bezogen auf die Gesamtheit der jeweils innerhalb dieser Grenzen - prinzipiell zur Vermittlung in Betracht kommenden Kommunikationspartner bzw. Kollektive und deren Mitteilungen muss geklärt werden, ob diese viel- bzw. möglichst allseitig zur Vermittlung gelangen oder nur partiell bzw. einseitig. Dies kann sinnvoll nur unter Berücksichtigung der Periodizität der Berichterstattung und beispielsweise anhand bestimmter Themen oder Themenkomplexe beurteilt werden. Prinzipiell denkbar sind auch Extremfälle, z. B. dass nur oder vorwiegend eine Gruppe in der vermittelten Kommunikation selbst „zu Wort kommt", andere Kommunikationsteilnehmer von diesen allenfalls zitiert werden ${ }^{14}$ (u. U. bei Parteimedien; hierbei kann die Integrationsleistung sehr hoch sein, aber beschränkt auf einen bestimmten Ausschnitt der Gesellschaft), oder dass Journalisten ausschließlich eigene Kommunikationsbeiträge (mit engerem oder weiterem Spektrum von Standpunkten), jedoch nicht die anderer gesellschaftlicher Akteure vermitteln. ${ }^{15}$

Im Hinblick auf die je einzelnen zu vermittelnden Mitteilungen stellt sich die Frage, ob diese bei der Bearbeitung durch die Vermittler prinzipiell gleich behandelt und ob die ursprünglichen Mitteilungen der jeweiligen Sprecher adäquat vermittelt werden, also trotz notwendiger Konzentration und mediengerechter Transformation gemäß dem ursprünglichen Sinn bzw. der Argumentation des Sprechers. Relevante Kriterien bzw. Vermittlungsregeln der Gleichbehandlung sind vor allem die Trennung von Berichterstattung und journalistischem Kommentar (so könnte bei mangelnder Trennung z. B. die Mitteilung eines Sprechers unkommentiert für sich stehen, die eines anderen verknüpft mit relativierenden Kommentierungen vermittelt werden u. Ä.) sowie

13 Man kann hier auch von speziellen und universellen Massenmedien sprechen (vgl. Wagner 1978: 201/202), welche Integrationsleistungen entweder für gesellschaftliche Subsysteme oder das Gesamtsystem erbringen.

14 So genannte „zitierte Partner“ (Wagner 1978: 97).

15 Auch in diesem Fall müsste die Integrationsleistung nicht unbedingt gering sein, vorausgesetzt die Journalisten machten sich zum Sprachrohr einer Vielzahl von Akteuren bzw. Kollektiven und deren Standpunkten, Wissensbeständen etc., ohne dabei die Darstellungen einseitig, aus einer bestimmten Sicht unterschiedlich zu werten bzw. unterschiedlich adäquat wiederzugeben; siehe folgende Ausführungen. 
die Transparenz der Sprecher ${ }^{16}$ und die Kennzeichnung ihrer Repräsentanzbeziehungen. ${ }^{17}$ Für die Einschätzung einzelner Mitteilungen sowie die Orientierung der Bürger innerhalb des Kommunikationsgeschehens und ihre eigene Positionierung darin ist diese Transparenz der Sprecher und ihrer Gruppenzugehörigkeit bzw. ihrer Repräsentanzbeziehungen von ganz erheblichem Wert (vgl. dazu ausführlicher Schönhagen 1998: 277ff.). Mangelnde Integrationsleistungen der Medien können deshalb auch darin bestehen, dass die Herkunft vermittelter Mitteilungen nicht offen gelegt oder verschleiert wird und so Repräsentanzbeziehungen unklar bleiben oder gar nicht zustande kommen.

Bei den genannten Kriterien handelt es sich um die wesentlichen Eckpunkte des traditionellen Unparteilichkeitskonzeptes des Journalismus (vgl. Schönhagen 1998). Letztlich kann man anhand der Analyse, wie parteilich bzw. unparteilich Medien vermitteln - unabhängig davon, ob dies Strategie ist oder eher unbewusst geschieht -, detaillierte Aussagen über deren Integrationspotenzial machen, wobei man diese Leistung natürlich in Relation zum jeweiligen Medientyp (siehe oben) setzen muss.

Jarren bezieht die angestrebte Analyse von Integrationsleistungen v. a. auf die Organisationsverfassung der Medien sowie auf das „redaktionelle Programm“ (2000: 38). Damit ist meines Erachtens vor allem die Vermittlung der sozialen Kommunikation in den Bereichen Politik, Wirtschaft, Kultur etc. gemeint, auf Medieninhalte bezogen also die so genannte „Information“ bzw. die Berichterstattung im weiteren Sinne sowie Talkformate. Zweifellos sind die genannten Gebiete für die Frage gesellschaftlicher Integration durch Kommunikationsvermittlung der entscheidende Untersuchungsgegenstand. Man sollte aber bedenken, dass auch Unterhaltungs-Inhalte wie Spielfilme, Serien, Kindersendungen, Musikprogramme etc. einen nicht zu unterschätzenden Beitrag zur gesellschaftlichen Integration leisten können; das oben kurz unter dem Stichwort Unparteilichkeit skizzierte Konzept und das im Überblick vorgestellte Massenkommunikationsmodell mögen dafür auf den ersten Blick, außer bei Talk-Formaten, weniger geeignet erscheinen. Die einzelnen Kriterien oder Fragen, insbesondere die nach Vielfalt oder allseitiger Vermittlung sowie die der Gleichbehandlung aller Urheber von Mitteilungen, lassen sich jedoch auch sinnvoll an die genannten Inhalte aus dem Bereich der Unterhaltung herantragen. So könnte man hier nach dem Spektrum der vermittelten Stile, Formen, Weltsichten, (Sub-) Kulturen, Bevölkerungsgruppen und dergleichen fragen (konkret z. B. nach den Geschlechtsrollenmustern in Zeichentrickfilmen für Kinder), welche letztlich ebenso Interpretationen von „Welt“, Standpunkte u. Ä. von Kollektiven darstellen bzw. zum Ausdruck bringen wie verbale Äußerungen oder Mitteilungen im engeren Sinne.

Folgt man allen diesen Überlegungen, dann ist für die empirische Analyse des Integrationspotenzials der Medieninhalte bzw. der Berichterstattung die Einbeziehung des vermittelten Kommunikationsprozesses entscheidend. Mit den üblichen Inhaltsanalysen lassen sich jedoch nur Aussagen über den Vermittlungsprozess machen: Man kann ermitteln, über welche Themen und Akteure (allgemein im Sinne von Handelnden, von Objekten der Berichterstattung) wie umfangreich, mit welchen Bewertungen etc. von

16 D. h. der Primärquellen, diese sind zu unterscheiden von den Vermittlungsquellen wie Nachrichtenagenturen u. Ä.; siehe dazu auch Punkt 3.

17 Dies geschieht normalerweise über bestimmte Repräsentanzmerkmale, die Hinweise auf die Herkunft des Sprechers und seine organisations- sowie kompetenzbezogene Legitimation etc. geben; siehe dazu auch Punkt 3. 
Journalisten berichtet wird. Folgende zentrale Fragen, die alle darauf abzielen zu klären, wen die vermittelten Selbstverständigungsdiskurse integrieren oder nicht, können damit jedoch nicht beantwortet werden: welche Sprecher aus der Gesellschaft in der Berichterstattung „zu Wort kommen“18 und welche Kollektive, Institutionen etc. sie dabei mit welcher Legitimation repräsentieren, ob nur Sprecher mit hohem Organisationsgrad „auftreten“ und wie umfangreich die einzelnen Standpunkte jeweils vermittelt werden, ob es dabei auffällige Einseitigkeiten bzw. Bevorzugungen oder Benachteiligungen gibt, die Sprecher neutral oder verquickt mit Kommentierungen durch die Journalisten vermittelt werden, und ob es latent bleibende Standpunkte gibt.

\section{Ein erweitertes Konzept der Inhaltsanalyse}

Auf der Basis des oben dargestellten Münchner Theorie-Ansatzes wurde ein spezifisches Inhaltsanalyse-Verfahren entwickelt ${ }^{19}$, das die Beantwortung der genannten Fragen zum vermittelten Kommunikationsprozess zu beantworten vermag und die Analyse des Vermittlungsprozesses zugleich mit umfasst. Ich beschränke mich bei der Darstellung der Methodik auf die Analyse von Berichterstattung im oben angesprochenen Sinne; konkrete Beispiele entnehme ich einer entsprechenden Inhaltsanalyse zur Wirtschaftsberichterstattung von Detlef Schröter. ${ }^{20}$ Es kann hier nur ein erster Überblick über die relativ komplexe Anlage einer solchen Inhaltsanalyse gegeben werden, zur Vertiefung sei auf die genannten Publikationen und die dortigen Code-Pläne verwiesen.

Gemäß der Unterscheidung des gesellschaftlichen Kommunikationsprozesses von dessen Vermittlung wird diese erweiterte Inhaltsanalyse auf zwei Untersuchungsebenen durchgeführt: Die Beiträge bilden die Untersuchungseinheit auf der Vermittlungsebene,

18 Gemeint ist „zu Wort kommen“ im Sinne oben erläuterter vermittelter Kommunikation, nicht etwa nur im Sinne von (wörtlichen) Zitaten, die z. B. in den Inhaltsanalysen des Medien Tenor (Hrsg. vom Institut für Medienanalysen $\mathrm{GmbH}$ in Leipzig unter Leitung von Roland Schatz) erfasst werden.

19 Eine grundlegende Einführung in das Verfahren findet sich in einem - leider unveröffentlichten - Manuskript von Hans Wagner (vgl. 1977).

20 Vgl. Schröter 1995. Diese Studie ist leider nicht mehr aktuell, sie wurde 1984 an vier Tageszeitungen, einer Wirtschaftszeitung, vier Wirtschaftsmagazinen und einem Nachrichtenmagazin am Lehrbereich von Prof. Wagner in München durchgeführt. Die Wirtschaftsberichterstattung sowie die Medienlandschaft haben sich inzwischen natürlich stark verändert; für die Veranschaulichung der Methode ist die Studie trotzdem in besonderer Weise geeignet, da ihre Zielsetzung - die Untersuchung journalistischer Qualität - in eine sehr ähnliche Richtung geht wie die hier zur Diskussion stehende Analyse der medialen Leistungen zur Integrationskommunikation. Für die Untersuchung des Integrationspotenzials von Unterhaltungsbeiträgen der oben genannten Art müsste das methodische Instrument jeweils angepasst werden. Eine ganze Reihe weiterer exemplarischer Studien, u. a. zahlreiche unveröffentlichte Magisterarbeiten, sind auf der Basis dieses erweiterten Konzepts der Inhaltsanalyse zu unterschiedlichen Fragestellungen durchgeführt worden. Der Übersichtlichkeit halber beziehe ich mich im Text nur auf die Untersuchung von Schröter. Einblicke in die Methodik, ihre vielfältigen Anwendungsmöglichkeiten sowie zum Teil Ergebnisse auch im Hinblick auf die hier zur Diskussion stehenden kommunikativen Integrationsleistungen bieten u.a. folgende Arbeiten: Ragusa 1981; Wagner 1984; Nawratil 1987; Strack-Zimmermann 1987; Haas 1995; Nawratil 1996; Sommer 1996; Bauer 1998. 
die vermittelten Kommunikationsteilnehmer - wobei mehrere in einem Beitrag vermittelte Partner getrennt erfasst werden können - bilden die Partnerebene (vgl. Schröter 1995: 77) bzw. die Ebene der sozialen Kommunikation.

Die Analyse auf der Vermittlungs- bzw. Beitragsebene umfasst neben den üblichen formalen Kriterien wie Datum, Medientitel, Fundort des Beitrags (Ressort, Sendung etc.), Darstellungsform, Umfang, Bilder/Illustrationen etc. die Erfassung der Vermittlungsquellen ${ }^{21}$ und des Vermittlungsanlasses, der nach verschiedenen Ereignistypen unterschieden werden kann. ${ }^{22}$ Bei der Untersuchung der Vermittlungsleistung ist es zudem wichtig, zwischen Anteilen der Fremd- und Eigenvermittlung zu unterscheiden; daran wird auch erkennbar, ob Berichterstattung und Kommentar getrennt werden. Bei der Fremdvermittlung vermitteln Journalisten Gesprächsbeiträge anderer Sprecher, bei der Eigenvermittlung dagegen entweder ihre eigenen Beiträge oder aber Grundlagen zum Verständnis der Mitteilungen, etwa in Form von Lexikon- oder Hintergrundwissen, einer Vorstellung der Sprecher o. Ä. ${ }^{23}$ Handelt es sich um eigene Gesprächsbeiträge (auch wenn Journalisten dabei als „Sprachrohr“ für andere fungieren sollten; erkennbar an den Repräsentanzbeziehungen), so werden diese im Einzelnen auf der zweiten Ebene mit erfasst. Die Form bzw. der Umfang der „Journalistenbeteiligung“ kann gegebenenfalls noch näher bestimmt werden (vgl. Schröter 1995: 255ff.). Weitere Untersuchungskriterien sind denkbar; Schröter z. B. untersucht auch die Überschriftengestaltung sowie Bewertungen von Unternehmen auf Beitragsebene. ${ }^{24}$

Auf der Kommunikations- oder Partner-Ebene wird das Spektrum der vermittelten Kommunikationsteilnehmer mit ihren Repräsentanzbeziehungen ermittelt, was im Hinblick auf die Integrationsleistung besondere Relevanz hat. Hier ist zunächst der Status der Partner zu bestimmen: Es können Sprecher auftreten (Einzelne wie Kollektive, Organisationen, Institutionen etc.; dieser „Organisationsgrad“ wird ebenfalls erfasst), andere Sprecher von diesen zitiert werden (welche demnach nur indirekt und von diesen quasi instrumentalisiert zu Wort kommen) sowie Angesprochene (ebenfalls Einzelne, Gruppen etc.) benannt werden. Hinsichtlich der Sprecher stellt sich dabei die Frage, ob diese genannt oder anonymisiert werden bzw. ungenannt bleiben. Im Hinblick auf das Integrationspotenzial entscheidend sind vor allem die folgenden Aspekte der Analyse:

21 Ausprägungen können z. B. sein: unbestimmbar, Redaktion bzw. Journalist, Nachrichtenagenturen, Dienste; weitere Differenzierungen sind natürlich möglich, außerdem können mehrere Vermittlungsquellen getrennt erfasst werden.

22 Schröter (vgl. 1995: 102ff.) unterscheidet zwischen Publikations-, Kommunikations- und Aktionsereignissen. Erstere sind schriftliche Vorlagen wie Berichte, Pressemitteilungen u. Ä. sowie vom Journalisten für die Publikation veranlasste Recherchen, Interviews u. Ä.; Kommunikationsereignisse sind Pressekonferenzen, Tagungen, Parlamentsdebatten u. Ä.; zu Aktionsereignissen zählen „Vorfälle und Unfälle“ (ebd.: 103), Streiks, Messen und ähnliche Anlässe.

23 Für Letzteres findet der Begriff der „Basalvermittlung“ (Schröter 1995: 131) Verwendung; es kann sich z. B. um eine kurze Zusammenfassung der Vorgeschichte handeln; vgl. dazu im Detail ebd.: $132 \mathrm{f}$.

24 Dies geschieht mithilfe von Polaritäten-Profilen, ähnlich den häufig bei Befragungen eingesetzten, wobei die Bewertungen durch Journalisten und durch andere Sprecher auf der Kommunikationsebene erfasst werden und damit unterscheidbar bleiben, auf Beitragsebene dann zusammengefasst werden (vgl. Schröter 1995: 79f.). 
- die Feststellung der Herkunft der Gesprächsteilnehmer, orientiert an einem vorher bestimmten „Kosmos“ möglicher Kommunikationsteilnehmer ${ }^{25}$ bzw. sachlicher wie geografischer Herkunftsbereiche;

- die Bestimmung der Repräsentanzbeziehungen der Gesprächsteilnehmer (soweit diese über rein statistische hinausgehen); diese werden anhand verschiedener $\mathrm{Va}$ riablen ermittelt: der organisationsbezogenen Legitimation (eine Ausprägung wäre z. B. die unbegrenzte Vertretungsbefugnis, wie etwa bei Vorsitzenden gegeben); der Kompetenzlegitimation (z. B. Fachspezialist), allerdings nur, wenn diese bezüglich des angesprochenen Sachverhalts relevant ist („Kompetenzrelevanz“); oder der Beanspruchung von Repräsentanz für einen bestimmten Herkunftsbereich;

- die Analyse der jeweiligen „Sprechmöglichkeiten“ (Schröter 1995: 78) der vermittelten Partner, von der Dokumentation im vollen Wortlaut bis hin zur knappen $\mathrm{Zu}$ sammenfassung ohne direkte oder indirekte Rede (vgl. im Detail ebd.: 266); ergänzend können der Umfang der Sprecher-Beiträge und die Häufigkeit ihrer „Auftritte“ im Beitrag gemessen werden; außerdem kann ermittelt werden, ob der Kommunikationspartner innerhalb des Beitrages seine Rolle tauscht, z. B. erst Sprecher, dann Angesprochener ist;

- die Untersuchung der Themen, zu denen die Kommunikationsteilnehmer sprechen bzw. angesprochen werden, orientiert an einem zuvor ermittelten „Kosmos“ potenziell denkbarer Themenbereiche oder -aspekte (Themen-Universalität);

- Bewertungen der Kommunikationspartner untereinander oder durch den/die Journalisten.

Ergänzend zur hier skizzierten Inhaltsanalyse sollte in einem weiteren Untersuchungsschritt die Frage geklärt werden, ob die jeweiligen Sprecher-Beiträge dem Sinn ihrer ursprünglichen Mitteilungen gemäß oder sinnentstellend vermittelt werden. Das kann entweder als qualitative Inhaltsanalyse anhand eines Vergleichs der vermittelten Konzentrate mit den ursprünglichen Mitteilungen der Sprecher geschehen, die in vielen Fällen ja vorliegen, oder wie bei Schröter (vgl. 1995: 156ff.) durch eine Befragung der Sprecher. Um den Aufwand überschaubar zu halten, kann in beiden Fällen eine derartige Analyse nur stichprobenartig an Fallbeispielen durchgeführt werden, wobei Sprecher unterschiedlicher Herkunftsbereiche, Organisationsgrade und Repräsentanzbeziehungen einzubeziehen sind.

\section{Resümee}

Im Hinblick auf die Einschätzung der massenmedialen Leistungen zur Integrationskommunikation kann mithilfe dieser erweiterten Inhaltsanalyse im Einzelnen geklärt werden:

- $\quad$ welche Kommunikationspartner und Kollektive - als Sprecher und Angesprochene, zitierte sowie repräsentierte Partner - in die vermittelten Selbstverständigungsdiskurse integriert werden und welche in der sozialen Kommunikation latent bleiben, inwieweit also der „Kommunikationsraum“ (Wagner 1978: 73), auf den das jeweils untersuchte Medium sachlich wie geografisch abzielt, tatsächlich von der Berichterstattung einbezogen wird;

25 Diese „Partneruniversalität“ (Schröter 1995: 77, 272) ist vergleichbar mit den auch sonst üblichen Themenuniversalitäten. 
- $\quad$ welche Themen und welche Perspektiven auf die Themen dabei berücksichtigt bzw. vernachlässigt werden;

- ob die vermittelten Kommunikationspartner gleichberechtigt in die vermittelten Diskurse integriert werden, oder ob es einseitige Bevorzugungen bzw. Benachteiligungen bestimmter Sprecher bzw. Kollektive oder gesellschaftlicher Bereiche bei der Vermittlung gibt, sei es durch unterschiedliche Sprechchancen, durch mangelnde Kennzeichnung der Partner und/oder ihrer Repräsentanzbeziehungen oder durch mangelnde Trennung von Nachricht und journalistischem Kommentar;

- ob nur oder vorwiegend gut organisierte Interessengruppen Zugang zur vermittelten Kommunikation finden und schlecht oder gar nicht organisierte Interessen unberücksichtigt bleiben, was ein Hinweis auf mangelnde Rechercheleistungen wäre; - welche Rolle die Journalisten bei der Vermittlung der gesellschaftlichen Diskurse sowie als Kommunikationspartner innerhalb derselben spielen, ob sie z. B. ihren privilegierten Zugang zur Vermittlung ausnutzen, um eigene Beiträge gegenüber denjenigen anderer Sprecher zu bevorzugen und damit möglicherweise ihre Vermittlungs- und Integrationsfunktion nur teilweise erfüllen, oder ob sie mit ihren eigenen Gesprächsbeiträgen stellvertretend bzw. anwaltschaftlich für andere gesellschaftliche Interessen das Wort ergreifen und für welche.

Im Ergebnis erhält man auf diese Weise Aufschluss darüber, inwieweit das untersuchte Medium für das thematisch bzw. sachlich wie räumlich definierte Gebiet, das es inhaltlich zu erschließen beabsichtigt, gewissermaßen als Forum ${ }^{26}$ dient oder, anders formuliert, inwieweit die Berichterstattung „sich um die Integration der real gegebenen Vielfalt der Interessenkonstellationen“ (Schröter 1995: 221) und der von den verschiedenen Seiten aktualisierten Themen bzw. Themenaspekte bemüht. Mit einer ergänzenden Befragung oder Fallstudie kann zudem ermittelt werden, wie adäquat die je einzelnen ursprünglichen Sprecher-Mitteilungen im Medium vermittelt werden, ob der Sinnzusammenhang erhalten bleibt, Zitate stimmen etc. Auch hierbei handelt es sich um eine zentrale Leistung zur Integrationskommunikation, da die Rezipienten nur bei adäquater Vermittlung die verschiedenen Positionen oder Perspektiven, wie sie von den Sprechern selbst in den gesellschaftlichen Diskursen dargelegt werden, nachvollziehen und sich selbst dazu (kommunikativ) in Beziehung setzen können. Außerdem können dabei erneut Bevorzugungen bzw. Benachteiligungen bestimmter Sprecher oder spezifischer Herkunftsbereiche von Sprechern sichtbar werden. Insgesamt kann so ermittelt werden, ob die unterschiedlichen Kommunikationspartner bzw. -Partnerschaften gerechte Zugangschancen zu den massenmedial vermittelten Selbstverständigungsdiskursen erhalten bzw. für welche und inwiefern dies nicht der Fall ist.

Eine solche, systematisch angelegte Analyse medialer Leistungen zur Integrationskommunikation könnte zum einen, wie bei Jarren deutlich wird, Grundlagen für kommunikationspolitische Fragen, Urteile und Maßnahmen bereitstellen, zum anderen auch den Rezipienten eine Orientierung in der Medienlandschaft im Hinblick auf die Vermittlungsleistungen einzelner Massenmedien ermöglichen. Angesichts der neueren Ent-

26 Der treffende und anschauliche Forumsbegriff findet in ähnlichen Zusammenhängen wie dem hier diskutierten seit einigen Jahren wieder häufiger Verwendung; so heißt es z. B. in der Entschließung des Europäischen Parlaments zur Rolle der öffentlichen Fernsehdienste in einer multimedialen Gesellschaft (1996: 653), diese sollten „ein Diskussionsforum für alle gesellschaftlichen Gruppen und Organisationen (...) bieten“. 
wicklungen in der Medienlandschaft muss man jedoch weiter fragen, welche Rolle den Massenmedien bzw. der Massenkommunikation - d. h. nach dem hier dargelegten Verständnis der von autonomen Vermittlungssystemen partnerunabhängig vermittelten sozialen Kommunikation - künftig zukommt. Verlieren universelle Massenmedien an Bedeutung, kann eine Vielfalt partikularer Massenmedien gesamtgesellschaftlich kommunikative Integration leisten? Welche Rolle spielt Massenkommunikation auch weiterhin innerhalb der computerbasierten Medien? Welche Rolle kann soziale Kommunikation ohne die Vermittlungs- und Konzentrationsleistung autonomer Vermittlungssysteme, wie sie in den „neuen“ Medien, insbesondere im Internet, in unterschiedlichen Formen zu beobachten ist, für eine kommunikative Integration der Gesellschaft spielen? Meiner Ansicht nach ist es zur Beantwortung dieser Fragen grundlegend, unterschiedliche Formen sozialer Kommunikation zu unterscheiden, die sich zweifellos auch in den computerbasierten Medien vollziehende Massenkommunikation (man denke an Online-Zeitungen, Webradio etc.) nicht zu vernachlässigen, und vor allem auch im Hinblick auf die neuen Kommunikationsmöglichkeiten nach Formen der Kommunikationsrepräsentanz zu fragen. ${ }^{27}$ Ohne diese ist eine möglichst allseitige kommunikative Integration, also eine Beteiligung prinzipiell jedermanns an den gesellschaftlichen Selbstverständigungsdiskursen in unseren komplexen Gesellschaften kaum realisierbar, zumal Voraussetzung einer Beteiligung ist, dass diese Diskurse für jedermann überschaubar bleiben.

\section{Literatur}

Aswerus, Bernd Maria (1993): Vom Zeitgespräch der Gesellschaft. Originaltexte, zusammengetragen von Hans Wagner. München.

Bauer, Markus (1998): PR-Erfolgskontrolle in der Pressearbeit. Ein Modell zur Überprüfung der kommunikativen Wirkung von PR-Argumenten. München.

Bohn, Cornelia (1999): Schriftlichkeit und Gesellschaft. Kommunikation und Sozialität der Neuzeit. Opladen/Wiesbaden.

Branahl, Udo (1996): Medienrecht: eine Einführung. 2. Aufl. Opladen.

Braun, Gabriele (1990): Massenmedien und Gesellschaft. Eine Untersuchung über die institutionelle Ordnung eines Kommunikationsprozesses freier Meinungsbildung. Tübingen.

Burkart, Roland (1998): Von verständigungsorientierter Öffentlichkeitsarbeit zum diskursiven Journalismus. In: Wolfgang Duchkowitsch u. a. (Hrsg.): Journalismus als Kultur. Analysen und Essays. Opladen/Wiesbaden, S. 163 - 172.

Entschließung des Europäischen Parlaments zur Rolle der öffentlichen Fernsehdienste in einer multimedialen Gesellschaft (1996). In: Media Perspektiven, Heft 12, S. 652 - 658.

Glotz, Peter (1990): Von der Zeitungs- über die Publizistik- zur Kommunikationswissenschaft. In: Publizistik, Heft 3, S. $249-256$.

Haas, Monika (1995): Angstpublizistik: Lebensmittelsicherheit. Wahnsinnsmeldungen - Die Berichterstattung über BSE. Eine Inhaltsanalyse. Unveröffentlichte Magisterarbeit. 2 Bde. München.

Hartmann, Frank (1996): Cyber.Philosophy. Medientheoretische Auslotungen. Wien.

Jarren, Otfried (2000): Gesellschaftliche Integration durch Medien? Zur Begründung normativer Anforderungen an die Medien. In: Medien E Kommunikationswissenschaft, Heft 1, S. $22-41$.

27 Leider kann darauf hier nicht näher eingegangen werden, da es sich um ausgesprochen komplexe Zusammenhänge handelt. Die Verfasserin beschäftigt sich mit diesen und weiteren Fragen zurzeit ausführlich in ihrem Habilitationsprojekt. 
Kepplinger, Hans Mathias (2000): Rezension zu: Philomen Schönhagen: Unparteilichkeit im Journalismus. Tradition einer Qualitätsnorm. Tübingen 1998. In: Medien E Kommunikationswissenschaft, Heft 3, S. 428/429.

Kohring, Matthias (2000): Rezension zu Ute Nawratil: Glaubwürdigkeit in der sozialen Kommunikation. Opladen 1997. In: Publizistik, Heft 2, S. $251-255$.

Krippendorf, Klaus (1994): Der verschwundene Bote. Metaphern und Modelle der Kommunikation. In: Merten, Klaus/Schmidt, Siegfried J./Weischenberg, Siegfried (Hrsg.): Die Wirklichkeit der Medien. Eine Einführung in die Kommunikationswissenschaft. Opladen, S. $79-113$.

Langenbucher, Wolfgang R. (1996a): Rezension von Hans Wagner: Journalismus I: Auftrag. Gesammelte Beiträge zur Journalismustheorie. Erlangen 1995. In: Publizistik, Heft 3, S. 357/358.

Langenbucher, Wolfgang R. (1996b): Auf der Suche nach den unbekannten Kommunikatoren. In: Aviso. Informationen der Deutschen Gesellschaft für Publizistik- und Kommunikationswissenschaft, Nr. 17, S. 7 - 10.

Langenbucher, Wolfgang R. (1997): WIR sind die KommunikatorInnen! Zu einigen Scheuklappen der Journalismusforschung. In: Bentele, Günter/Haller, Michael (Hrsg.): Aktuelle Entstehung von Öffentlichkeit. Akteure - Strukturen - Veränderungen. Konstanz, S. $19-38$.

Luhmann, Niklas (1996): Die Realität der Massenmedien. 2., erw. Aufl. Opladen.

Maletzke, Gerhard (1963): Psychologie der Massenkommunikation. Theorie und Systematik. Hamburg.

Marcic, René (1965): Öffentlichkeit als staatsrechtlicher Begriff. In: Nenning, Günter (Hrsg.): Richter und Journalisten. Über das Verhältnis von Macht und Presse. Wien, S. 153 - 228.

Nawratil, Ute (1987): „Wenig Lärm um viel“. Berichterstattung über Reproduktionsmedizin und Genforschung. Vergleichende Inhaltsanalyse. Unveröffentlichte Magisterarbeit. München.

Nawratil, Ute (1996): Glaubwürdigkeit in der sozialen Kommunikation. Mit Fallstudien zur Berichterstattung über politische Skandale. Dissertation, 3 Bde.; empirischer Teil bislang noch nicht veröffentlicht. München. (Theoretischer Teil 1997 erschienen unter dem Titel: Glaubwürdigkeit in der sozialen Kommunikation. Opladen/Wiesbaden.)

Neidhardt, Friedhelm (Hrsg.) (1994): Öffentlichkeit, öffentliche Meinung, soziale Bewegungen. Opladen.

Ragusa, Rosario (1981): Der Medien-Stiefel: Italienberichterstattung in der deutschen Presse. Frankfurt a. M.

Rühl, Manfred (1985): Integration durch Massenkommunikation? Kritische Anmerkungen zum klassischen Integrationsbegriff. In: Saxer, Ulrich (Hrsg.): Gleichheit oder Ungleichheit durch Massenmedien? Homogenisierung - Differenzierung der Gesellschaft durch Massenkommunikation. München, S. $19-32$.

Schönhagen, Philomen (1998): Unparteilichkeit im Journalismus. Tradition einer Qualitätsnorm. Tübingen.

Schönhagen, Philomen (1999): Der Journalist als unbeteiligter Beobachter. In: Publizistik, Heft 3, S. $271-287$.

Schröter, Detlef (1995): Qualität und Journalismus. Theoretische und praktische Grundlagen journalistischen Handelns. München.

Sommer, Annette (1996): Ein Fall von Political Correctness? Die Diskussion um die Islamwissenschaftlerin und Friedensnobelpreisträgerin Annemarie Schimmel in der Presse. Unveröffentlichte Magisterarbeit. München.

Stock, Martin (1985): Medienfreiheit als Funktionsgrundrecht: Die journalistische Freiheit des Rundfunks als Voraussetzung allgemeiner Kommunikationsfreiheit. München.

Strack-Zimmermann, Marie-Agnes (1987): Bilder aus Amerika. Eine zeitungswissenschaftliche Studie über die USA-Berichterstattung im Zweiten Deutschen Fernsehen (ZDF). Frankfurt a. M. u. a.

Wagner, Hans (1977): Modell einer Inhaltsanalyse nach zeitungswissenschaftlichen Kriterien. Unveröffentlichtes Manuskript. München.

Wagner, Hans (1978): Kommunikation und Gesellschaft. Teil I: Einführung in die Zeitungswissenschaft. München. 
Wagner, Hans (1980): Rationalisierungsprozesse der Sozialen Kommunikation. Materialien zu einem besseren Verständnis der Massenkommunikation. In: Politische Bildung, Heft 1, S. 3 - 32.

Wagner, Hans (1984): Wirklichkeits-Präparate? Das Medikamenten-Bild in deutschen Print-Medien. In: Wagner, Hans/Starkulla, Heinz (Hrsg.): Medizin \& Medien. Krankt die Gesundheit am Journalismus? München, S. $10-104$.

Wagner, Hans (1988): Vermittlungsverfassung in der Massenkommunikation. Zeitungswissenschaftliche Theorie der journalistischen und publizistischen Darstellungsformen. In: Wolfgang. R. Langenbucher (Hrsg.): Publizistik- und Kommunikationswissenschaft. Wien, S. 182 $-189$.

Wagner, Hans (1993): Das Fach-Stichwort: Zeitungswissenschaft - eine Wissenschaft vom Menschen. In: Aswerus, Bernd Maria: Vom Zeitgespräch der Gesellschaft. Zusammengestellt und eingeführt von Hans Wagner. München, S. 121 - 197.

Wagner, Hans (1995a): Das Fachstichwort: Massenkommunikation. In: Otto Groth: Vermittelte Mitteilung. Ein journalistisches Modell der Massenkommunikation. Hrsg. von Wolfgang R. Langenbucher. München, S. 187 - 240.

Wagner, Hans (1995b): Journalismus I: Auftrag. Gesammelte Beiträge zur Journalismustheorie. Erlangen 